\title{
Optical Fibre Sensors: a Current Perspective
}

\author{
Brian Culshaw*
}

University of Strathclyde, Glasgow, Scotland, UK

\begin{abstract}
This paper presents a very brief overview of the principles which have evolved and the achievements which have accrued over almost half a century of fibre optic sensors. We cite a few examples of success stories along the way, but the very diverse nature of optical fibre sensor technologies of necessity infers that these few examples will be but representative and many are omitted. We also speculate on the future evolution of fibre sensors, both in terms of applications potential and the incorporation of research into 'new' optics into the fibre sensor platform.
\end{abstract}

Keywords: Fibre sensors, applications environment, future prospects.

\section{INTRODUCTION}

Optical fibre sensor technology has been with us approaching half a century. Over that period it has, in common with every other specialised sensing technology, established a number of application niches into which the unique properties of fibre sensing offer unique solutions to difficult measurement problems. However, unlike the majority of specialised sensor technologies, it has maintained a surprising level of interest and excitement among the research community who continue to discover innovative approaches to realising novel sensing and measurement concepts, many of which will be described in this special issue.

The unique benefits of fibre optics are well rehearsed but are worth repeating. They include the fact that the sensor itself is electrically passive and the links to and from the sensing region are totally immune to electromagnetic interference and are electrically insulating. They also feature the very attractive prospect of addressing over 100 individual sensor elements in a completely electrically passive spatially scattered array extending over many kilometres, and the unique capability to make distributed measurements through which a measurand map may be obtained along the length of the fibre up to ranges which can exceed 100 kilometres. Additionally, the technology addresses optical measurements directly. This is an obvious observation but is also at the heart of the immense range of measurement parameters which can be addressed using the guided wave fibre optic medium coupled to appropriate optical and electronic signal interpretation [1-3].

It all started with the Fotonic ${ }^{\mathrm{TM}}$ sensor patented in mid 1960 's as a means for measuring displacement and surface roughness. The sensor, the very simple principles of which are shown in Fig. (1), is still available as a commercial surface roughness measuring instrument. The emergence of single mode fibres around 40 years ago opened up the pros

*Address correspondence to this author at the Strathclyde University, Glasgow, Scotland, UK; Tel: +44 1505610 349;

E-mails: b.culshaw@ee.strath.ac.uk, brianculshaw@yahoo.co.uk pects for interferometric sensing with early demonstrations of fibre optic gyroscopes and hydrophones emerging in the mid to late 1970 's. Even these, however, have at least some of their roots in conceptual publications a decade earlier [4]. The ideas of sensor networks [5] and distributed sensing (Fig. 2) began to emerge 30 years ago as interest grew in the prospects for using non-linear phenomena, Raman and Brillouin scatter as a basis for performing distributed signal measurements [6].

The purpose of this paper is to endeavour to provide some background for the research papers which follow many of which build upon this early work. With this in mind, we shall quickly summarise the operating principles which underline most, possibly all, fibre optic sensor technologies and examine a few examples of how some of these technologies have matured into practical operational sensor systems. To provide a backdrop on how things might evolve in the future, we briefly look into the overall applications environment and potential markets and attempt to put these into the contexts of future sensing configurations exploiting emerging photonic technologies and addressing expanding market and application needs.

\section{THE BASIC PRINCIPLES}

Optical fibre sensors are, overall, based upon two generic architectures - intrinsic and extrinsic (Fig. 3). In the former the fibre itself provides the transduction between the parameter to be measured and the modulation of the light within the fibre. In the latter, this transduction process takes place in some form or other of gap between the feed and return fibre connections. The light source ranges from relatively high peak power single spatial and temporal mode lasers to very broad band light sources which are essentially spatially incoherent. At the receiver end of the system, the photodetector converts the optical intensity received into a voltage and this voltage hopefully represents the parameter which the system is designed to measure and this parameter alone. The basic physics of all the processes involved does, however, limit even the potential viability of this assumption to temperature measurements and even in this limiting case the stability of 


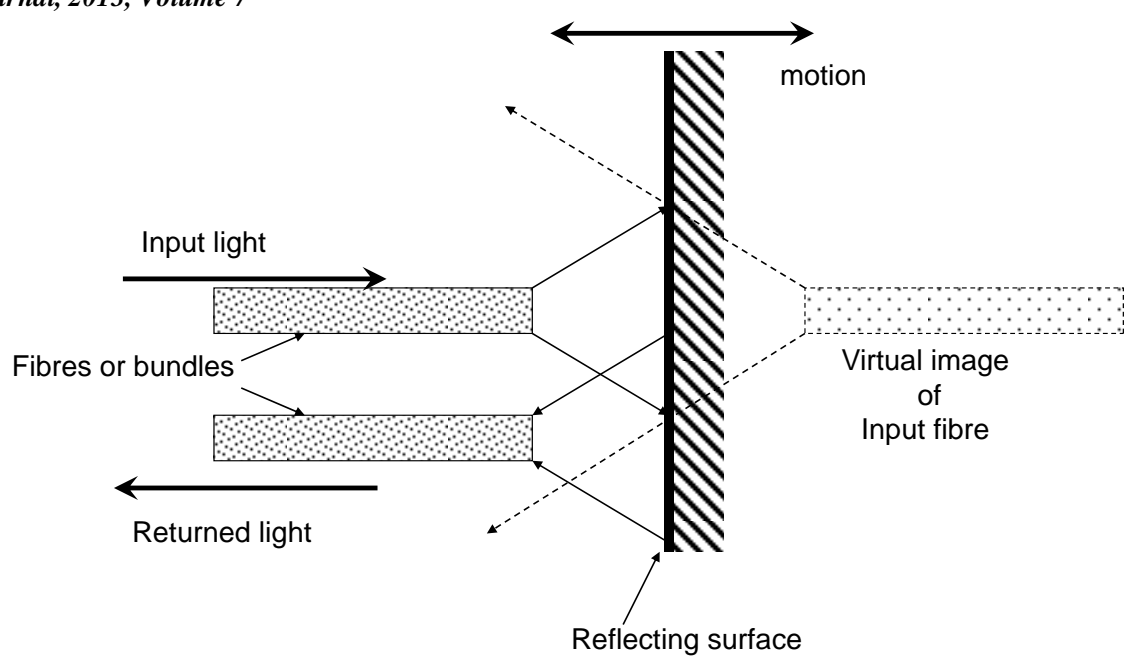

Fig. (1). The essential elements of the 'FotonicTM sensor (http://www.mtiinstruments.com/products/mti2000fotonic.aspx). The position of the reflecting surface with respect to the fibre end is determined by the reflected optical power, normalised using a second reference collection channel (Menadier, Kissinger and Adkins, Instrum. and Control Systems, 40,p114 1967)
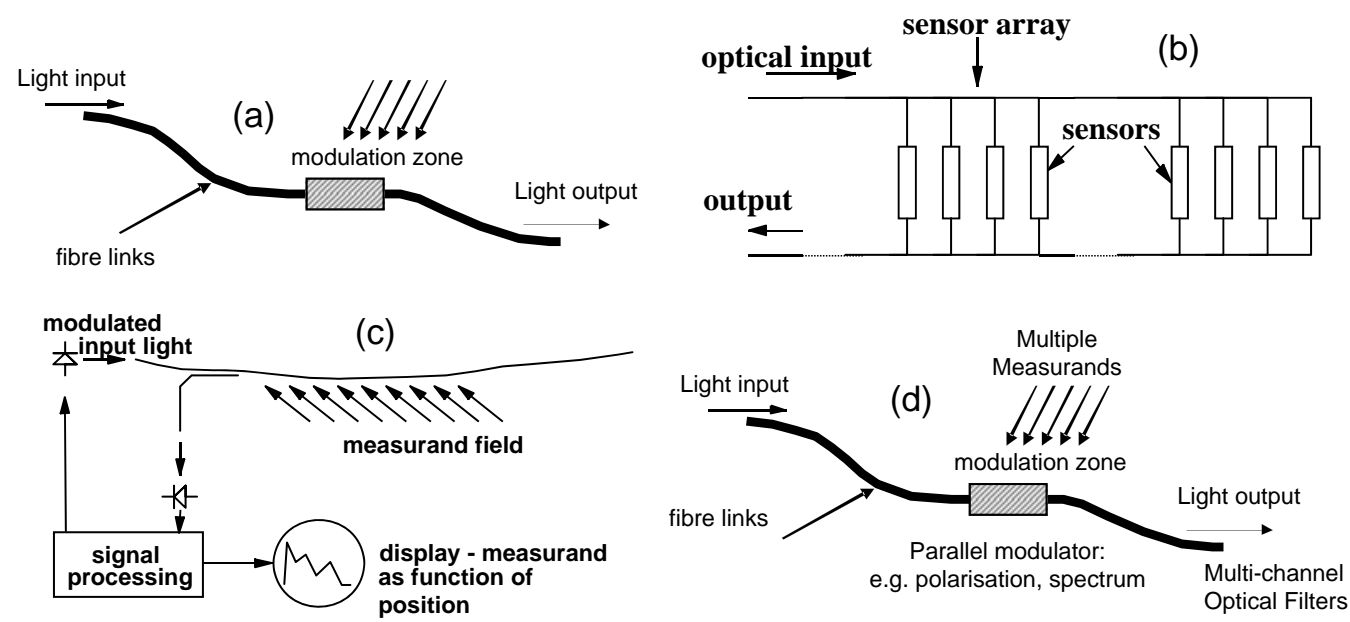

Fig. (2). The architecture options for optical fibre based sensor systems. (a) single sensor, (b) sensor array, (c) distributed sensor and (d) multiparameter sensor
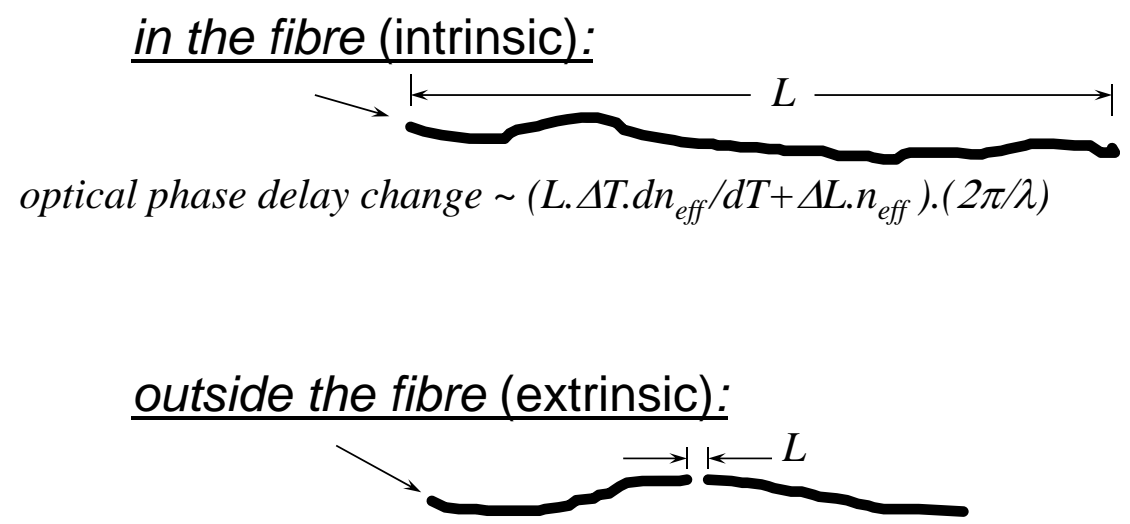

Same relationship, but now applied to material in the gap

Fig. (3). Examples of intrinsic (the light remains in the fibre from source to detector) and extrinsic (the light emerges from the fibre into a modulation zone) fibre optic sensors, here applied to the interferometric measurement of distance changes.

the receiver itself can sometimes cause significant drift. This very simple discussion highlights one of the principal operational compromises with fibre optic sensors and virtually every other sensing technology. They all respond to temperature.
Table 1 below attempts to encapsulate the essential optical parameters which can be modulated using fibre sensors, the measurands to which this modulation process may apply and the essentials of the corresponding detection processes. The details of these processes are described extensively in 
Table 1. Basic Approaches to Fibre Sensing and their Applications

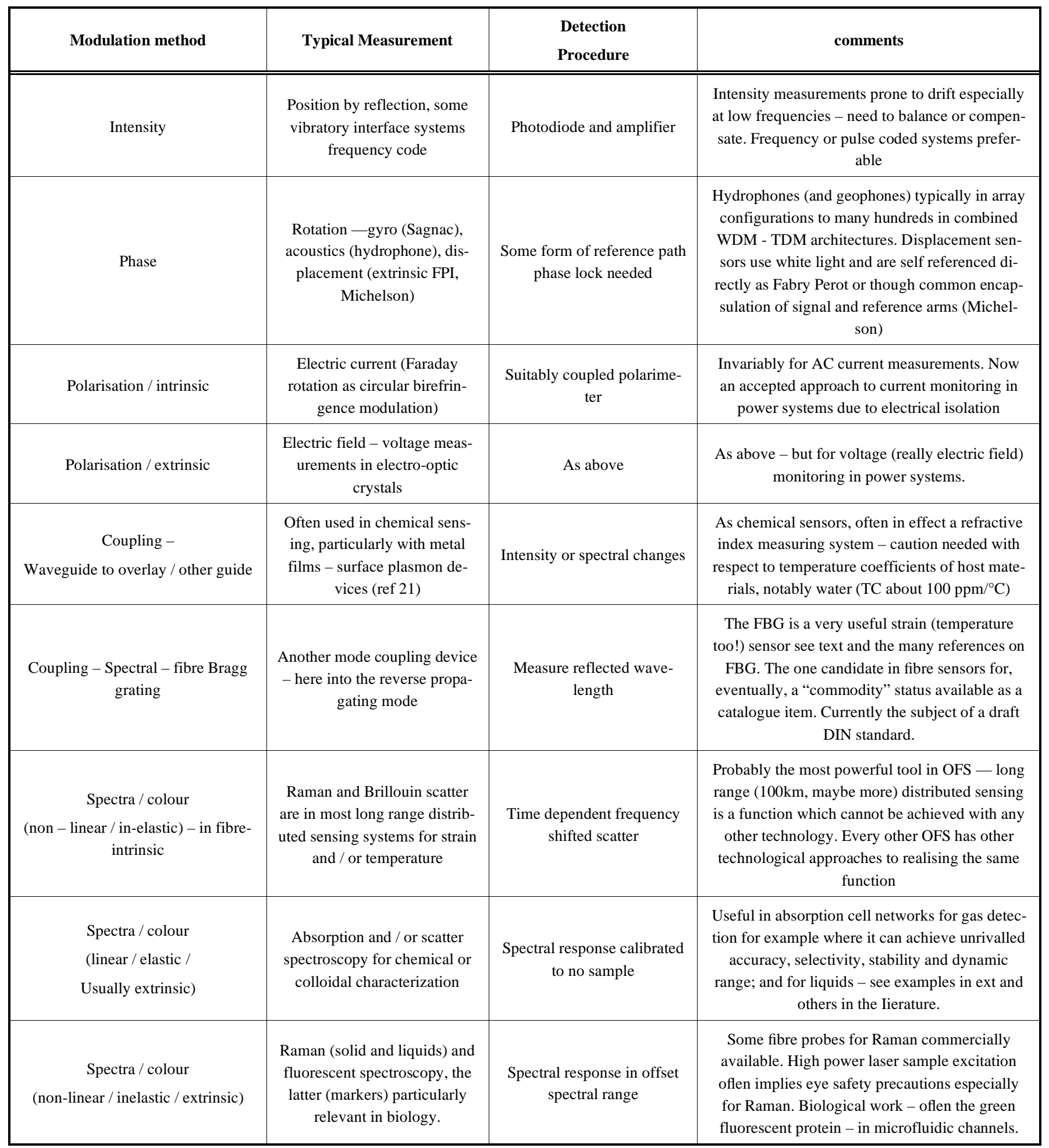

the literature and the aim here is simply to highlight the range of optical techniques and sensing architectures which may be addressed using fibre sensing technology.

Arguably the other principal generic feature of fibre sensors is the immense diversity of networking configurations which the basic technology facilitates. Fig. (2) attempted to summarise this. All these networks are electrically passive from the optical source to the photodiode receiver and the distance between the source and receiver can extend from centimetres to a hundred kilometres and more. It is these passive networks which are one of the principal operational advantages afforded by fibre sensor technology.

In addition to these basic technological principles which underpin all optical fibre sensor technologies, it is also per- 


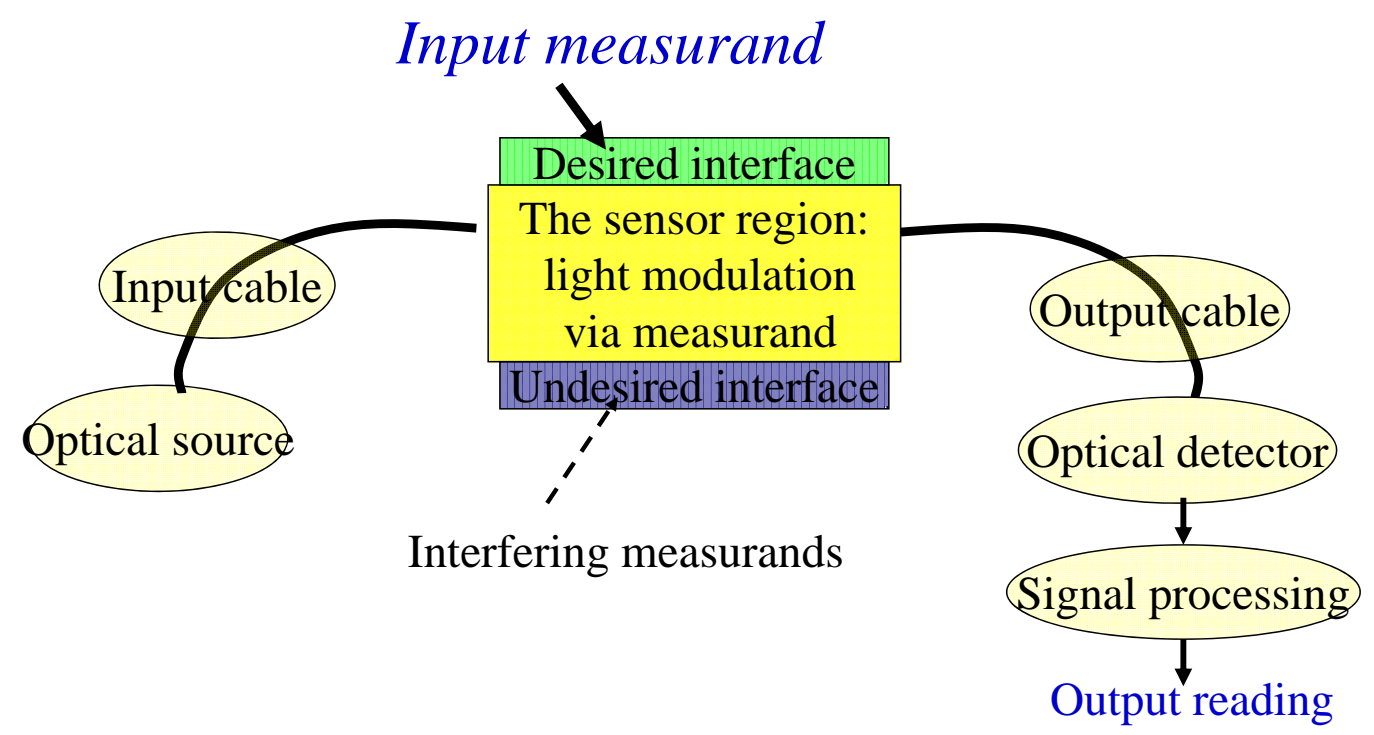

Fig. (4). The fibre optic sensor as a measurement tool...illustrating the interfering phenomena which distort the final perceived measurement. The output includes contributions from all the pale boxes and the interfering measurands in addition to the influence of the input measurand.

haps worth mentioning a few generic design and implementation issues. The first of these concerns the inherent drift characteristics of any detection system which converts optical intensity into electrical voltages. This conversion factor will vary, albeit by a small amount, with time and temperature and additionally will depend upon optical wavelength. Furthermore, the response function is unlikely to be linear with optical intensity over more than - say - a $20 \mathrm{~dB}$ dynamic range. Any sensing system which relies upon intensity modulation transduction between measurand and light must recognise this. Furthermore, since all sensing systems rely upon intensity detection, this dependence must also be recognised in sensor design. The second principal factor is the simple observation that everything depends upon temperature and most probably all transduction mechanisms between an applied measurand and the input to the final optical detector will within themselves be temperature dependent. This temperature dependence is sometimes relatively straightforward to filter out, for example vibration measurements usually occur in a temporal frequency range which is much higher than typical temperature variations. Sometimes, though, they are inextricable one from the other. For example some forms of chemical sensors, which in effect measure concentrations in water as changes in refractive index, do so in the presence of a temperature coefficient of index in water of around $100 \mathrm{ppm} /{ }^{\circ} \mathrm{C}$. In cases like this accurate temperature stabilisation of the sample is critical.

The basic principles of fibre sensing then comprise two important and complementary features. The first is the scientific and technological implementation of the modulation processes which relate the detected output signal voltage to the parameter at the input end which the sensor is designed to measure. The second and invariably much more difficult issue to resolve is that of ensuring that the parameter to be measured is, as closely as possible, uniquely related to the final output voltage and to critically identifying that this relationship is characterised and understood. Fig. (4) illustrates these many dependencies.

\section{THE TECHNOLOGY - SOME COMMENTS ON ITS CURRENT IMPLEMENTATIONS}

This section will briefly describe a few of the optical fibre technologies which have been adopted in practice or which promise to be adopted in some form or other in the near future. It will also explore the reasons why these particular realisations of fibre sensing have proved to be of interest in the user environment.

Distributed sensors are unique to optical fibre technologies. The basic architecture shown in Fig. (2) has, however, evolved into a slightly more complex configuration for the vast majority, possibly all, of the current applications. The complexity lies in the use of non-linear phenomena (inelastic scattering) as the basis for sensing (Fig. 5). For Raman scatter the ratio between the Stokes and Anti-Stokes scatter signatures at a particular wave number space from the excitation signal, is a unique function of temperature. This effect, first noted 30 years ago, has been very successfully implemented [7] into a range of distributed temperature sensing systems with applications as diverse as tunnel monitoring for the outbreak of fire to temperature profile assessment in operational oil wells. Stimulated Brillouin scatter has also been used as the basis of distributed sensing systems. Here it is the measurement of the frequency offset between the scattered signal and the carrier which is used as an indicator of, in fact, acoustic velocity within the optical fibre. The acoustic velocity is in turn a function of temperature and strain and in various implementations stimulated Brilllouin scatter has been used in extensive distributed measurement systems applied to, for example, long distance pipe lines and large mechanical structures [8].

More recently distributed sensing has developed into Rayleigh scatter based systems operating in a coherent detection mode. These have demonstrated intriguing performance parameters not only as intrusion detection systems but also as distributed microphones [9]. It seems that these are likely to find applications in, for example, perimeter fence security 


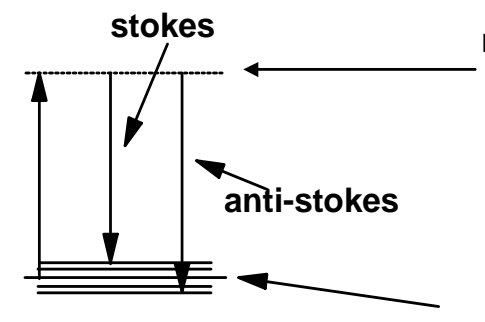

ground state (of fibre material - silica)

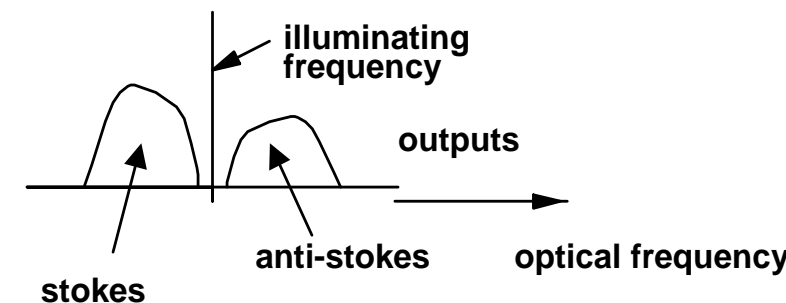

stokes metastable level

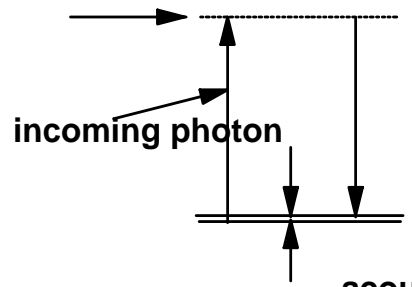

acoustic phonon energy

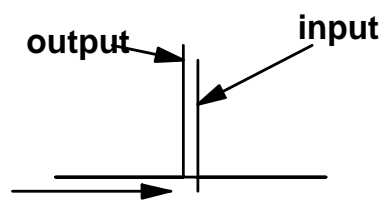

\section{BRILLOUIN}

Non-linear scattering processes in optical fibre

Fig. (5). In-elastic (non-linear) processes used predominantly in distributed sensing. Raman scatter can be used to reliably measure temperature profiles (and temperature alone) by taking the ratio of the Stokes and anti-Stokes power levels. Stimulated Brillouin scatter shown here gives acoustic velocity, a function of both temperature and strain.

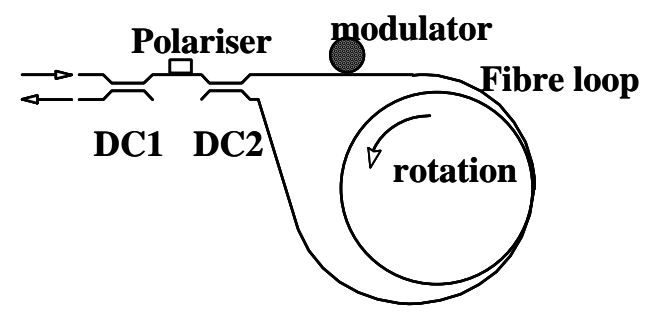

Architecture of the optical fibre gyroscope

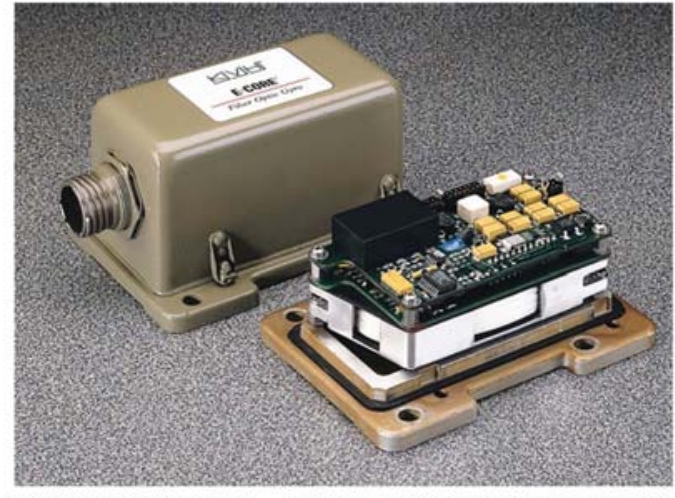

A fibre optic gyroscope - courtesy KVH, Tinley Park, III, USA

Fig. (6). The essential elements of the fibre optic gyroscope - a fibre implementation of the Sagnac interferometer, together with a photograph of one of the many practical realisations of such a system.

monitoring and possibly in covert surveillance. Distributed sensing is currently by far the dominant technology in terms of fibre sensor markets and is likely to retain this position over the coming years.

The fibre optic gyroscope was one of the earliest demonstrations of fibre sensor technology. It has evolved into a highly competitive approach to measuring rotation rates with an entirely different set of application benefits to those which we find in distributed sensing. The fibre optic gyroscope (Fig. 6) is compact, has no mechanical moving parts, its scale factor is fundamentally independent of acceleration forces and, provided the laser wavelength can be kept stable, its output is independent of temperature [10]. The fibre gyroscope is also an example of very careful, indeed rather subtle but also rather straightforward, optical system design required to ensure that these advantages were realised. The first fibre optic gyroscopes flew in commercial aircraft around 1997 some 20 years after initial demonstration. Since then they have found applications as diverse as antenna stabilisation and submarine navigation. The actual detailed technical requirements for each of these many and varied applications vary enormously as indeed do expectations in production volumes and per unit costs so that even within this one rather specialised operational principle there is a rich diversity in the actual realisation.

The fibre optic hydrophone was originally demonstrated at around the same time as the fibre optic gyroscope. However, the fibre hydrophone is attractive for an entirely different set of reasons. Here, for example, weight is a consideration since neutral buoyancy is far easier to achieve with fibre technology than the previously established piezo ceramic systems. However, most persuasive is the capability of the hydrophone to be configured in vast arrays of sensors extending over, if necessary, kilometres. The story is again a 


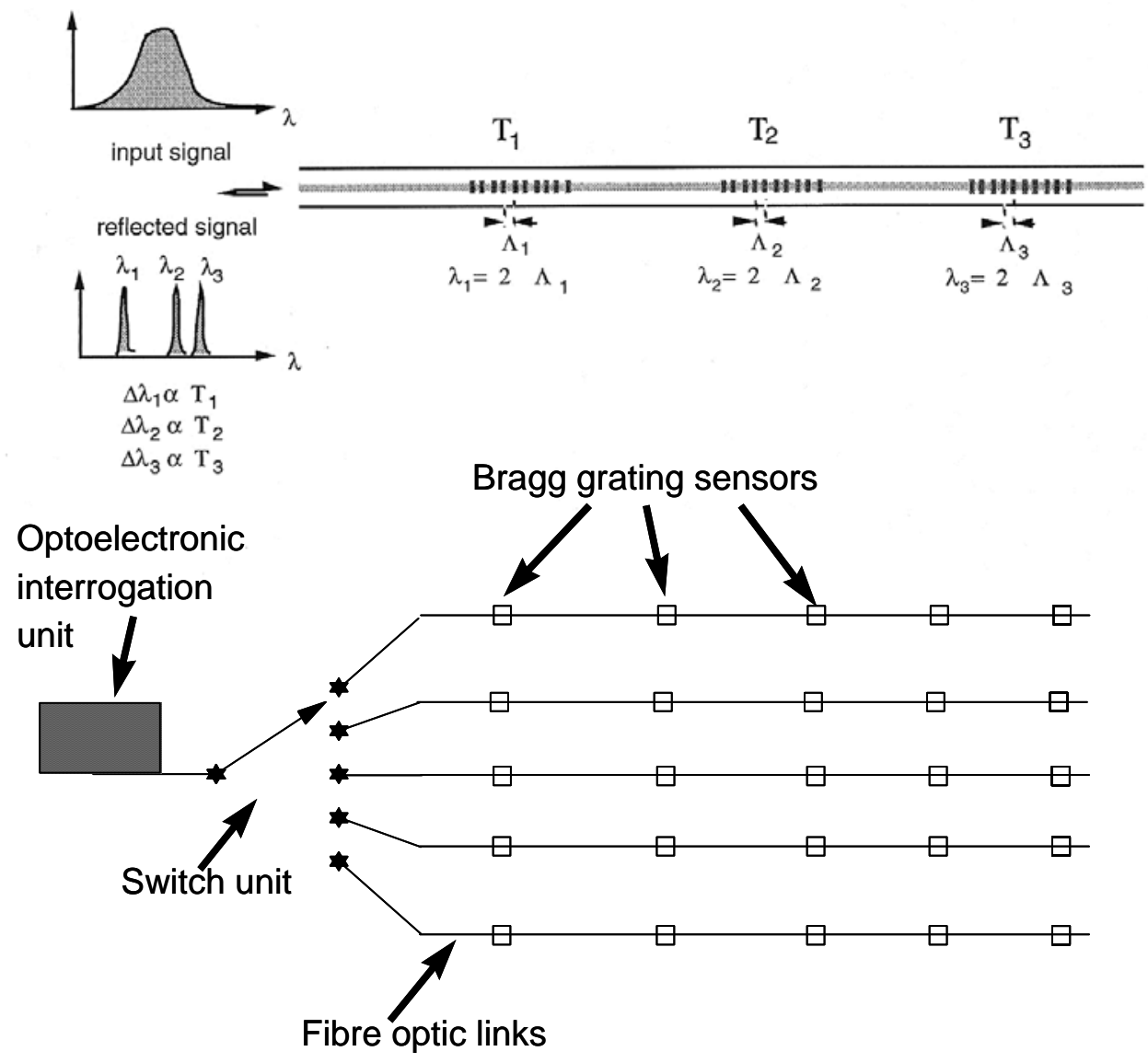

Fig. (7). Fibre Bragg Grating arrays for very large passive sensor systems. Reflection wavelengths depend on the optical length of the grating - a combination of temperature and strain effects. There can be many tens of gratings on a single fibre and there have been numerous system realisations especially in structural monitoring applications.

long one but currently an array of approximately 20,000 sensors is being installed for seismic surveying purposes on the bed of the North Sea [11].

All of the above have been intrinsic sensors for which a length of fibre acts as the transduction medium. The hydrophone and the gyroscope along with the Rayleigh backscatter intrusion systems are all, in effect, interferometers whilst the other distributed sensors rely upon non-linear optical phenomena. The other thus far relatively successful intrinsic sensing systems have exploited fibre Bragg gratings (Fig. 7). The wavelength reflected from a Bragg grating sensor is a function of the optical path characterising each grating period and this in turn depends on temperature (through thermal expansion and more so through the refractive index variation with temperature) and strain. The fibre grating has arguably made its greatest impact in its role as a sensing element for structural monitoring in large structures such as wind turbines and bridges [12, 13]. Here the benefits are simple (relatively) installation procedures and material compatibility coupled to the all optical electro-magnetically passive network capability. In applications such as wind turbines any thermal variations can easily be filtered out since vibrational signals are almost invariably occur at the turbine rotational frequency. In the example of wind turbines the vibrational signals have been used to provide input to control systems to optimise the blade orientation and the angle of the generator system with respect to the prevailing wind. Fibre gratings have also been used in specialised structural evaluation projects associated with, for example, electric trains [14] where they have successfully monitored the mechanical response of new pantograph designs and vehicle induced movement of newly installed railway tracks.

Chemical analysis is another potential application sector for optical fibre sensing though here, with a few exceptions, the extrinsic architecture is more appropriate - the fibre acts as a light source to remotely illuminate a chemical sample and collect the light modified after its passage through the sample. The interaction mechanisms between incident light and sample are potentially numerous but the most important spectroscopic processes include both absorption and scatter spectroscopy and non-linear phenomena such as fluorescence and Raman spectroscopy. All these processes can be applied either to the sample itself or to some form or other of the intermediate chemical indicator. The term "spectroscopic measurements" is in fact a generic wavelength dependent measurement of the complex refractive index so that the refractive index approaches are a subset of these measurements.

Conventional spectroscopy with suitable data interpretation can be very effective. Systems such as the one illustrated in Fig. (8) measuring the wavelength dependence of both direct absorption and scattering signals can be ex- 

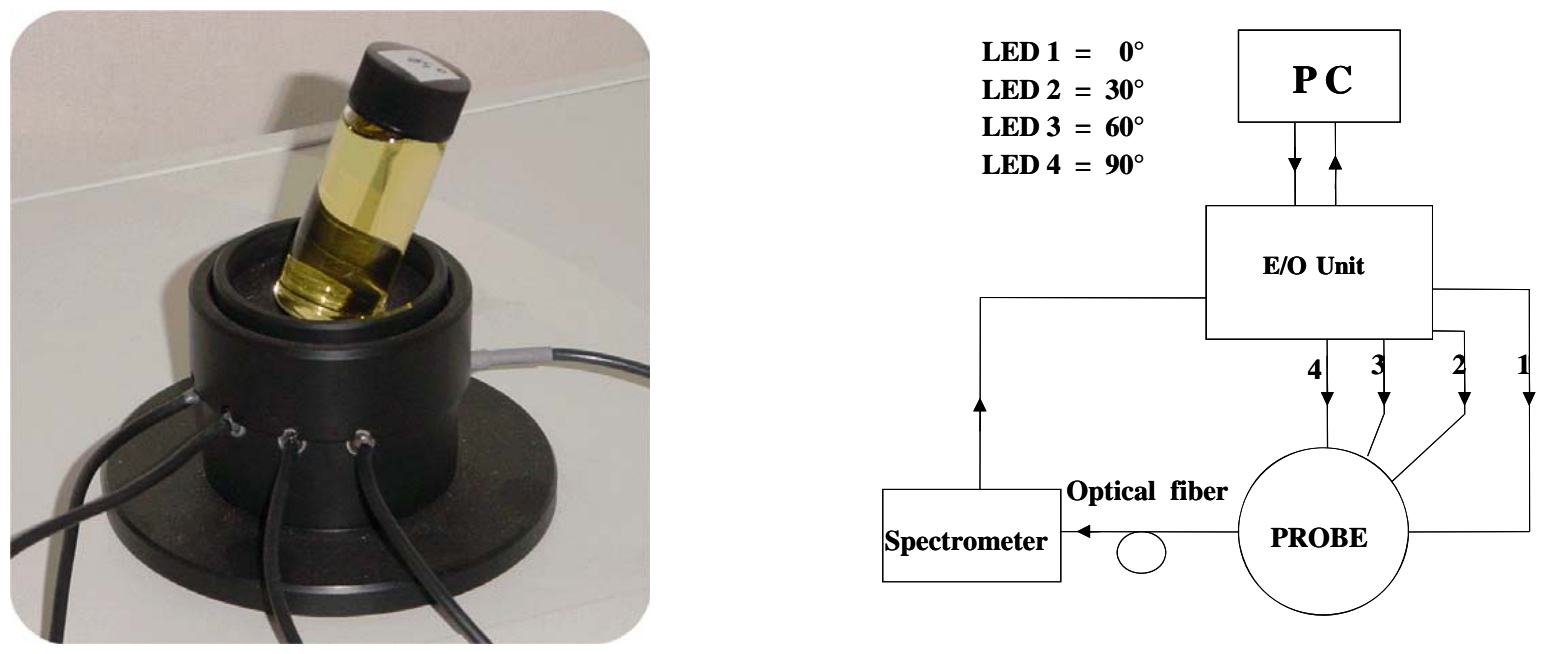

Fig. (8). The elements of so called spectral nephelometry though which the transmission and scattered spectral responses of a sample are measured. Left - the laboratory equipment and right a schematic diagram of the apparatus. (Courtesy Anna Mignani, CNR IFAC, Florence, Italy).
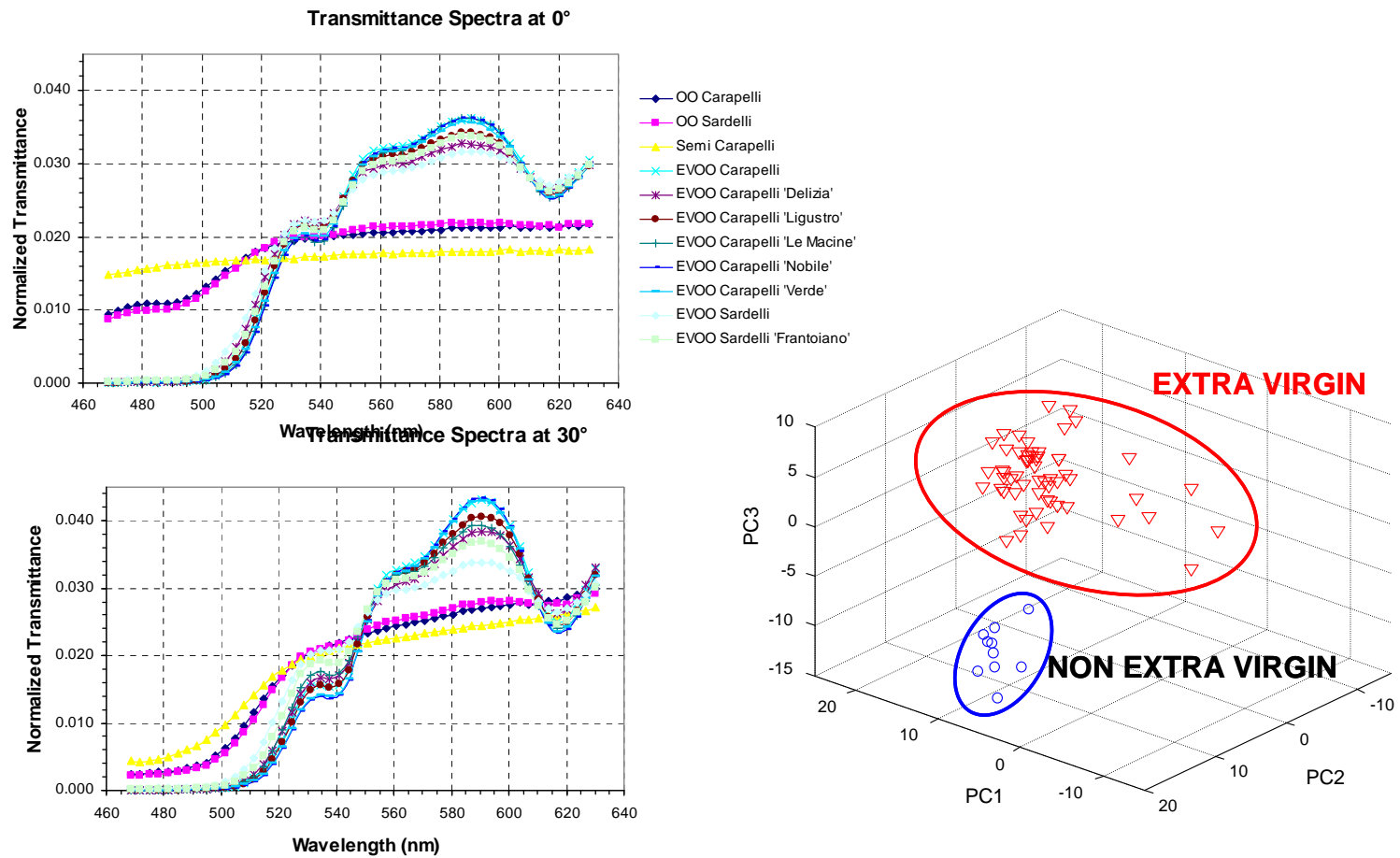

Fig. (9). Some typical absorption (upper) and scatter at $30^{\circ}$ spectra for olive oil samples and right - the results of a principal components analysis on this experimental data demonstrating the discrimination ability inherent in the technique.

tremely powerful. Fig. (9) shows some measurements on olive oils using this technique. These measurements after careful processing are a reliable discriminator among the numerous regional variations of extra virgin oil and also between extra virgin and non-extra virgin categories [15].

Spectroscopy can in addition be a useful tool for gas measurements since many gas species have absorption bands within the $1-2 \mu \mathrm{m}$ wavelength region over which optical fibres are particularly transparent. Using tunable diode lasers as the optical source facilitates the realisation of complex networks (Fig. 10) capable of detecting, for example methane gas at concentrations compatible with explosive gas monitoring application [16]. Furthermore with signal processing suitably applied such systems are also capable of measuring the temperature and / or pressure of the gas species within the detection cell over distances of kilometres.

This very brief account of a few examples of the application prospects of fibre sensors highlights a number of generic important features. The first is that each application is very specific and exploits different aspects of the fibre sensor technology in often very different physical and environmental surroundings. Furthermore, the actual technology used in the optical domain also encompasses a considerable range. The second point is that all require very considerable 


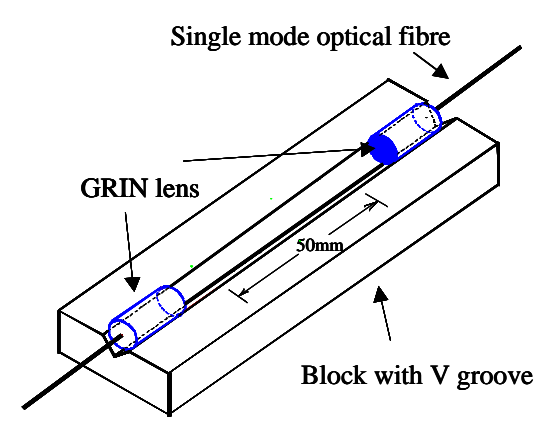

- No electrical power outside
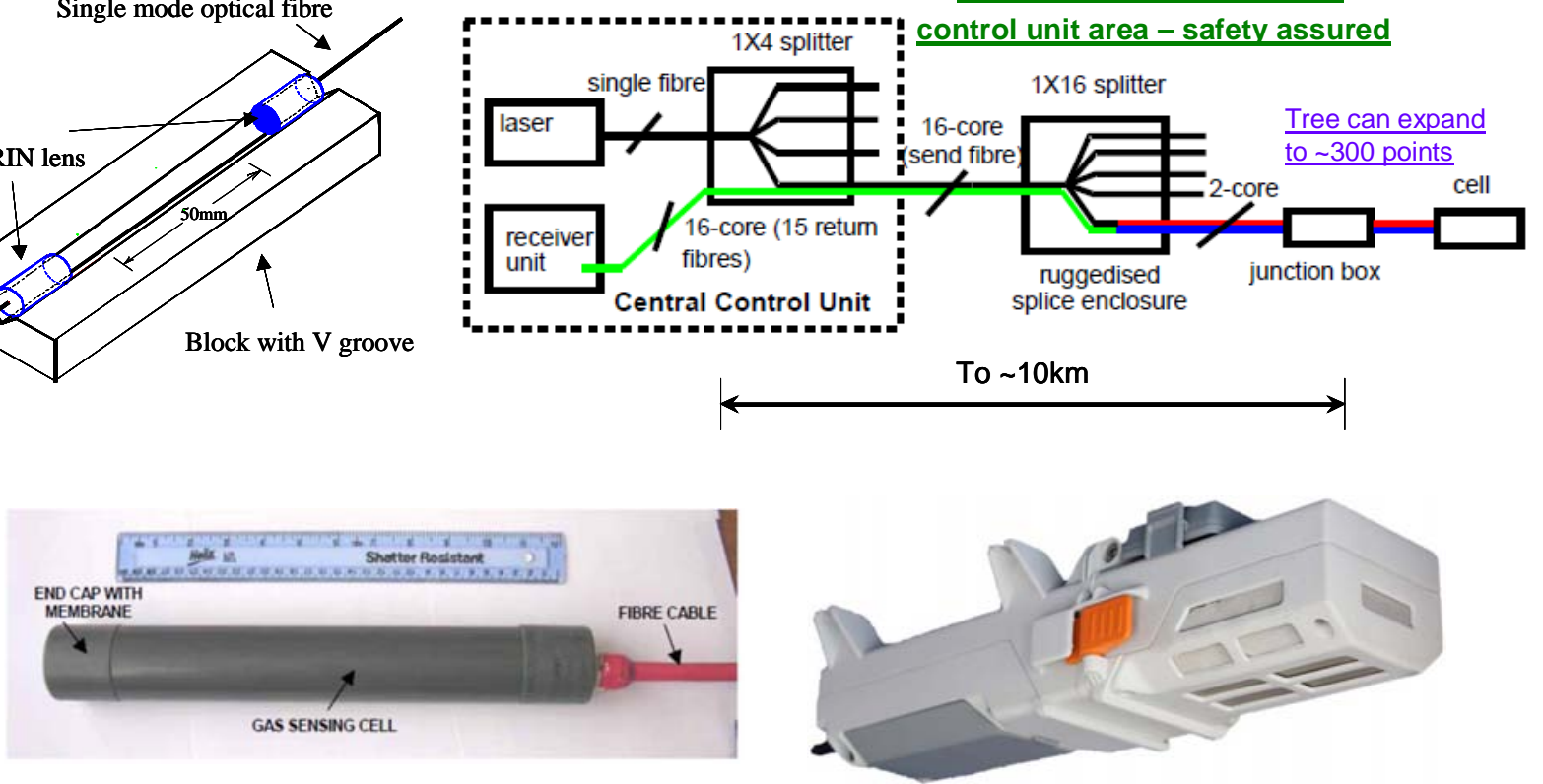

Fig. (10). Gas spectroscopy using fibre optics - top left a sketch of a typical absorption cell and top right a full network diagram. Below, some practical absorption cells, both of the order $40 \mathrm{~cm}$ in linear dimensions (OptoSci)

attention to technical issues other than optics. Perhaps the most important of these other issues concern packaging and environmental protection, electronic system design and development and frequently comprehensive data processing development in order to produced useful user information from the reams of data which the sensor systems often produce. This is immediately obvious in the case of the spectral measurements of olive oils but is also critically important in all distributed fibre optic sensing systems and in the interpretation of data from large networks. Of course, in addition to resolving the technical issues the sensor engineers must also establish user confidence and ensure that communications between the users, sensor engineers and, very frequently, some form of certification authority are effectively established and maintained. Fibre optic sensing is then far from a simple single homogenous endeavour and requires multi disciplinary approaches to ensure its successful implementation.

\section{SOME COMMENTS ON THE APPLICATIONS EN- VIRONMENT AND MARKETS}

The researchers' perspective on fibre sensing is quite appropriately to evaluate new techniques for using guided wave optics to make measurements of the physical, chemical and biological world around us. However even the simplest demonstration should acknowledge the context of what has gone before and is already available, both from fibre technologies and elsewhere, and should endeavour to critically assess how well this new sensing approach can actually perform the task in hand. This critical assessment should always encompass the sensitivity to interfering measurands both of the same category (for example closely related chemical species in chemical sensing) and totally unrelated to the measurement in hand such as vibration and temperature. Another important trade-off is the balance between the usefulness of the measurement which is enabled and the difficulty in real- ising the measurement system itself. Furthermore, most measurements of interest have already been made using some other technique so the question of how well the prospective fibre solution performs compare to the established routine is clearly very important and for the eventual acceptance of the fibre technique. Persuasive discussion around this point is absolutely essential. There are often many tens, sometimes hundreds, of different approaches to making the same measurement.

Of course, there are some measurements which have not been made but it would be useful were they to become available. Distributed measurements in tunnels and pipelines exemplify this and the benefits compared to hundreds or thousands of discrete monitoring points are immediately obvious. Even here a very close relationship between the user and supplier is essential to establish confidence in something which has never before been available.

And then there are specifications and protocols and all the very important, but for the average research worker somewhat outside the mainstream, aspects of operational methods, standards, environmental testing and established practice which are all part and parcel of the user interaction with the eventual technology provider. This interaction, which may well come under the overall theme of market development, typically consumes far more highly skilled effort than the technology itself. Careful and effective marketing for specialised systems like fibre sensors is crucial.

And the projected market figures emphasis the need for these relationships. The total current fibre optic sensor market is variously presented at figures around the $\$ 1 \mathrm{~B}$ per annum for the global total, of which something in the region of $70-80 \%$ is distributed sensor systems. The current projections do, however, give rise to some optimism putting the figure at around $\$ 3 \mathrm{~B}$ per annum in around 5 years time and, even after allowing for the need for the market survey industry to sell re- 


\section{Distributed Optical Fibre Sensors Market - predicted 2006}

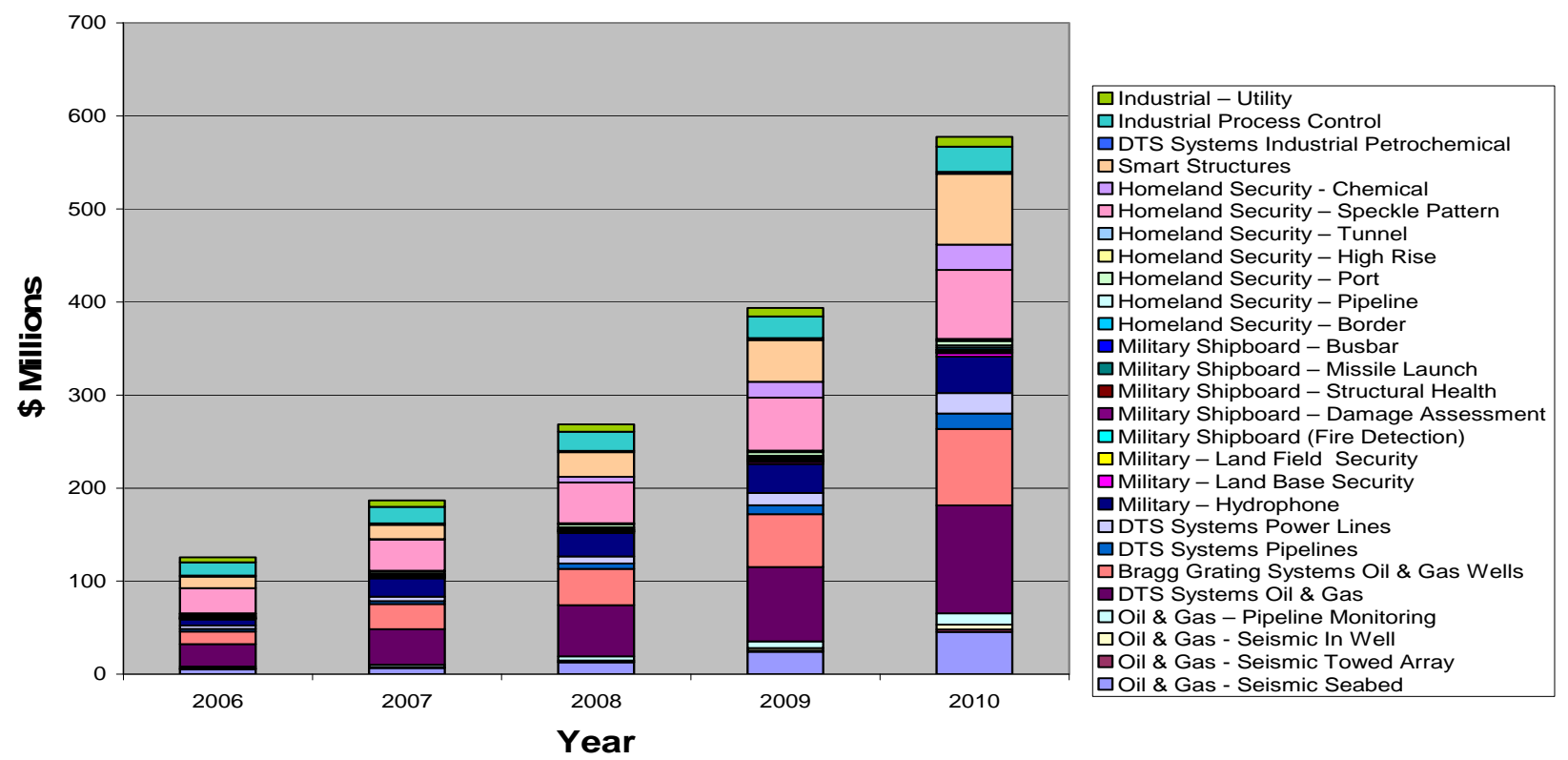

Fig. (11). A market prediction for distributed sensors (around $80 \%$ of the total) made through OIDA in 2006. The predictions were reasonably good, but the real message lies in the very many sectors into which distributed sensing is applied. Each sector ahs significantly different technical requirements, even for nominally similar technologies.

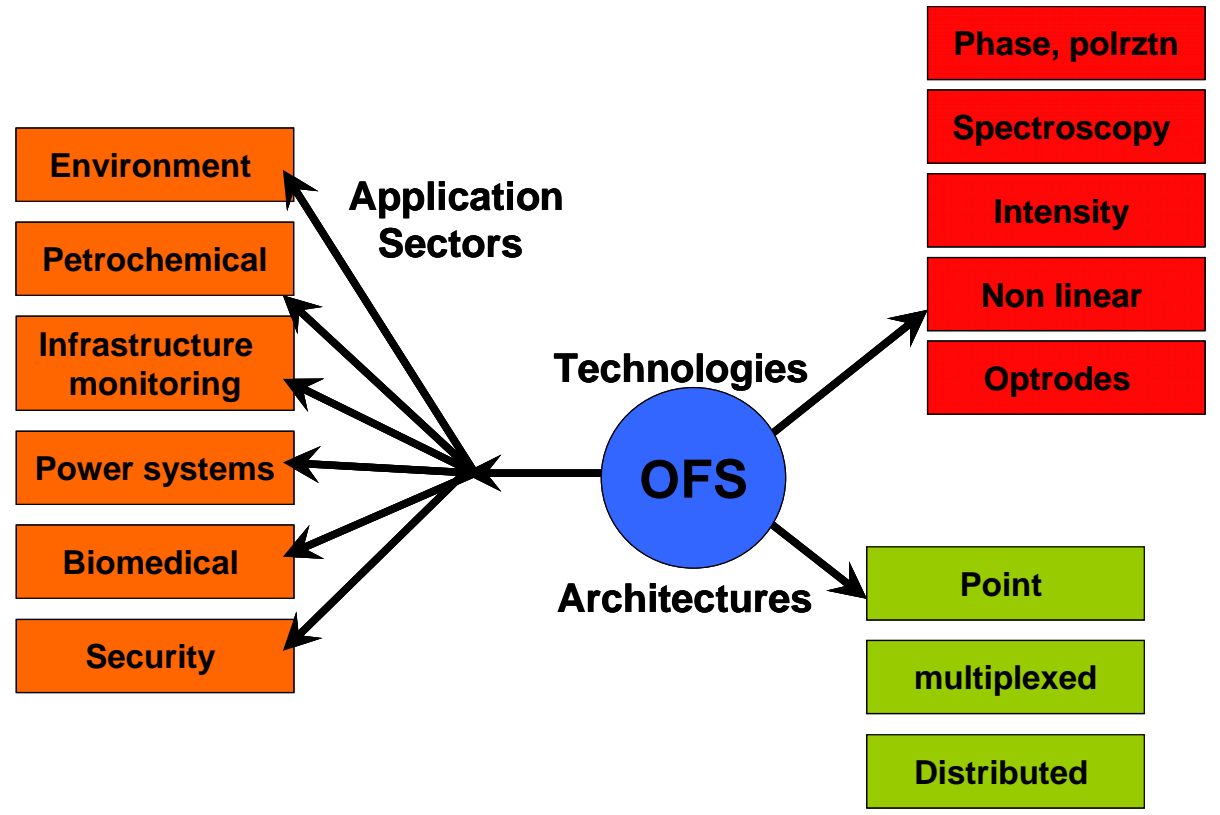

Fig. (12). Illustrating the very fragmented nature of optical fibre sensors, in terms of optical modulation technology, system architectures and broad applications sectors. Each combination of application, modulation approach and network architecture imposes a (often very) different set of constraints.

ports, there is certainly a very positive message within these numbers. They are inevitably accepted by everyone to be simply guidelines - even the market numbers which operate retrospectively and tell us how much the market was a year or two ago. The immense fragmentation of the market is also evident and the Fig. (11) shows some (now retrospective) projections which highlight this fragmentation and emphasise that even within this relatively modest total for a global endeavour there are multiple specialised fragments within this total. These, in turn, link inextricably to the highly frag- mented industry, comprising predominantly small companies, which typifies current fibre sensor activity.

That said the young, optimistic, energetic, entrepreneurial photonics engineer with an interest in fibre sensing has every reason to anticipate an interesting future.

\section{AND WHAT ABOUT THE FUTUTRE}

Here we turn to speculation and where and how fibre sensing might progress in the coming years given that the 
- Make solar energy economical

- Manage the nitrogen cycle

- Advance health informatics

- Prevent nuclear terror

- Advance personalised learning

- Provide energy from fusion

- Provide access to clean water
- Engineer better medicines

- Secure cyberspace

- Engineer the tools of scientific discovery

- Develop carbon sequestration methods

- Restore and improve urban infrastructure

- Reverse engineer the brain

- Enhance virtual reality

Fig. (13). Engineering's grand challenges according to the National Academy of Engineering US (http://www.engineeringchallenges.org/) Areas in which fibre sensors could make a significant contribution are highlighted.

technologies, the markets and the applications are (Fig. 12) immensely diverse, so there is obviously great scope for future research endeavours.

New technologies are becoming available through which we shall see greater control of optical signals and inevitably this greater control will find some application in the sensor domain. Already we have seen photonic crystal fibres being assessed and evaluated as sensors, though as yet, despite the 15 year history, little has emerged of practical consequence apart from the very broad bandwidth, single spatial mode "white light" source [17]. Undoubtedly this will find, in time, extensive use in sensing applications. Polymer fibres too have been with us for even longer but recently, thanks to the emergence of single mode structures and reduced losses, have excited interest in the sensing community to whom their mechanical flexibility offers intriguing measurement potential [18].

The increasing availability of precision micro and nano optics will surely also offer prospects in sensing enabling unprecedented capabilities in the manipulation and precision location of optical signals to, in principle, sub wavelength precision. It is always curious that whilst sub wavelength resolution is often an anathema to the optical engineer, to the radio frequency engineer it has been forever accepted without question.

There are other tools too which will doubtless facilitate more and possibly better measurement systems including the ever increasing diversity in optical sources and detectors, the ability to manipulate the optical properties of materials through, for example, quantum dots and related concepts and ever increasing precision capability in mechanical assembly - when single mode fibre was first mooted many thought of it as a non-starter because of the difficulty in precision alignment for connectors. Then, of course, there is all that we can do with electronic signal processing both at the input end to the system in terms of control of light sources and its temporal and spectral parameters and the rich diversity of tools through which the output results can be filtered to highlight features which really are of interest.
It is, however, possibly in the application space that the greatest opportunities lie. There are obvious pressing sensing and measurement requirements in environmental monitoring and atmospheric assessment, in safety and security systems, in assessing large areas and in measuring the very small, the very confined and the inaccessible. Many of these applications will consolidate as the lobbyists' wish lists (or at least some of it) evolves into the politicians' legal framework. This is particularly the case in sensitive issues such as climate change, water quality control, pollution monitoring and safety and security assessment. There are undoubtedly emerging prospects for the appropriately enhanced fibre optic tool box in these sectors. In the broader context the US National Academy of Engineering a few years ago produced their "grand challenges" which are still current and are summarised in Fig. (13) on which we have highlighted just some of the areas within which fibre sensing could contribute.

So the future for fibre sensors presents opportunities for research and product innovation at the technological level and applying an ever expanding technological tool box to every more challenging societal issues. This all forms a part of an overall optimistic outlook for photonics as "the $21^{\text {st }}$ century electronics" and recent reports published from the EU (The 2nd Photonics Research Agenda, February 2010) [19] and the United States (Optics and Photonics - Essential Technologies for our Nation, August 2012) [20] emphasise that the outlook for photonics engineering is indeed optimistic.

\section{CONFLICT OF INTEREST}

The authors confirm that this article content has no conflicts of interest.

\section{ACKNOWLEDGEMENTS}

The author has benefitted significantly from countless interactions with students at Strathclyde and elsewhere, with research consortia, with industrialists and with sensor users. He has had the good fortune to have worked with and interacted with the community both nationally and internationally. 


\section{REFERENCES}

[1] Lopez-Higuera JM, Eds. Handbook of optical fibre sensing technology. NY: Wiley 2002.

[2] Culshaw B, Dakin JP, Eds. Optical fiber sensors. MA: Artec House 1988-1997; pp.1-4.

[3] Udd E. An overview of optical fiber sensors. Rev Sci Instrum 1995; 66(8): 4015

[4] Snitzer EJ. Phase modulation in optical fibres. IP Patents 3, 10; $1182,1960$.

[5] Dakin JP, Wade CA. Optical fibre hydrophone array - recent progress. In: Culshaw B, Ed. Optical sensor multiplexing systems, Proc sensors and systems ' 82 . Proceedings of the $2^{\text {nd }}$ International Conference on Optical Fibre Sensors; Stuttgart; Buckingham UK: Pasadena Ca. Network Publications 1984; pp. 375-379.

[6] Culshaw B. Distributed and multiplexed fibre sensor systems. In: Chester AN, Martelluci S, Scheggi AM, Eds. Optical fibre sensors. Martinus Nijhoff: Dordrecht 1987; pp. 165-84.

[7] Dakin JP, et al. Distributed optical fibre temperature sensor using a semiconductor light source and detector. Electron Lett 1985; 21:569-70.

[8] Horiguchi T, Karashima T, Tateda M. Tensile strain dependence of Brillouin frequency shift in silica optical fibres. IEEE Photon Technol Lett 1989; 5: pp.107-8.

[9] Juarez JC, Maier EW, Choi KN , Taylor HF. Distributed fiber optic intrusion sensor system. IEEE JLT 2005; 23(6): p. 2081.

[10] OFS 22 to be held in Beijing in October 2012 will dedicate a special workshop to the $35^{\text {th }}$ anniversary of the fibre optic gyroscope. Proceedings will be published by SPIE

[11] Kringlebotn JT, Nakstad H, Eriksrud H. Fibre optic ocean bottom seismic cable system: from innovation to commercial success. Pro- ceedings SPIE $750320^{\text {th }}$ International conference on optical fibre sensors; 2009 October; Edinburgh

[12] Culshaw B. Fibre optic sensors in smart structures: achievements, challenges and prospects. Proceedings SPIE, Vol7982, Smart Structures and Materials, San Diego, March 2011.

[13] See for example, papers from the workshop on Industrial Progress, Commercial Systems and Field Tests in Proc OFS 22 Beijing October 2012. (Proceedings to be published by SPIE)

[14] Ecke W, Schroeder K, Kautz M, et al. On line characterisation of impacts on electric train current collectors using integrated optical fibre grating sensor network. Proceedings of SPIE 2005; 5758 , Smart Structures and Materials.

[15] Mignani AG, Ciaccheri L, Gordillo B, et al. Indentifying the production region of single malt Scotch whiskies using optical spectroscopy and pattern recognition techniques'. Sens Actuat B: Chem 2012; 172; pp. 458-62.

[16] Stewart G, Johnstone W, Thursby G, Culshaw B. Near infrared spectroscopy for fibre based gas detection. Proceedings of SPIE Orlando Florida; 2010; vol. 7675.

[17] Dudley JM, Genty G, Coen S, et al. Supercontinuum generation in photonic crystal fibres. Rev Mod Phys 2006; (78), p. 1135.

[18] Proceedings POF 2011 Bilbao Spain Session SN1, POF papers in OFS 22 and also K Krebber, 'Smart technical textiles based on fibre optic sensors', to be presented OFS 22 Beijing October 2012 (Proceedings to be published by SPIE).

[19] Second Strategic Research Agenda in Photonics European Technology Platform Photonics 21 January 2010. available at: http://cordis.europa.eu/fp7/ict/photonics/docs/reports/ph21-sra2_en.pdf

[20] Optics and Photonics: Essential Technologies for Our Nation. National Academies Press (USA). 2013; available at: http://www.nap.edu/catalog.php?record_id=13491

(C) Brian Culshaw; Licensee Bentham Open.

This is an open access article licensed under the terms of the Creative Commons Attribution Non-Commercial License (http://creativecommons.org/licenses/by-nc/3.0/) which permits unrestricted, non-commercial use, distribution and reproduction in any medium, provided the work is properly cited. 